NEIP-99-004

gr-qc/9903066

Int. J. Mod. Phys. A (in press)

\title{
DISTANCE MEASUREMENT AND $\kappa$-DEFORMED PROPAGATION OF LIGHT AND HEAVY PROBES
}

\author{
Giovanni Amelino-Camelia ${ }^{a}$, J, Jerzy Lukierski ${ }^{b}$ and Anatol Nowickic ${ }^{c}$, \\ ${ }^{a}$ Institut de Physique, Université de Neuchâtel, \\ rue Breguet 1, CH-2000 Neuchâtel, Switzerland \\ ${ }^{b}$ Institute for Theoretical Physics, University of Wrocław, \\ pl. M. Borna 9, 50-204 Wrocław, Poland \\ ${ }^{c}$ Institute of Physics, Pedagogical University, \\ pl. Słowiański 6, 65-029 Zielona Góra, Poland
}

\begin{abstract}
We investigate the implications for the measurability of distances of a covariant dimensionful " $\kappa$ " deformation of $D=4$ relativistic symmetries, with quantum time coordinate and modified Heisenberg algebra. We show that the structure of the deformed mass-shell condition has significant implications for measurement procedures relying on light probes, whereas in the case of heavy probes the most sizeable effect is due to the nontrivial commutation relation between threemomenta and quantum time coordinate. We argue that these findings might indicate that $\kappa$-Poincaré symmetries capture some aspects of the physics of the Quantum-Gravity vacuum.
\end{abstract}

\section{Introduction}

One of the aspects of Quantum Gravity that has been most actively investigated is the possibility that there be a minimum uncertainty for the measurement of distances. The simplest and best understood proposal is the one of a "minimum length"

$$
\Delta x \geq L_{\min },
$$

which fits well the expectation of certain studies [2, 3] of measurability in quantum gravity. Eq. (1.1) might also hold in critical sting theory as suggested by analyses of

\footnotetext{
${ }^{1}$ Supported by a grant of the Swiss National Science Foundation

${ }^{2}$ Partially supported by KBN grant 2P03B130.12
} 
string collisions at Planckian energies, which were found [4 to be characterized by the following modified uncertainty relation

$$
\Delta x \geq \frac{\hbar}{\Delta p}+\alpha G \Delta p
$$

where $G=c^{2} l_{p}^{2} / \hbar$ is the gravitational coupling (Newton) constant, $c$ and $l_{p}$ are the speed-of-light and Planck-length constants respectively, and $\alpha$ is a constant related to the string tension (Regge slope). Clearly Eq. (1.2) implies $L_{\min } \sim \sqrt{\hbar \alpha G}$.

While modified uncertainty relations are not necessarily associated to quantum groups [3], it is interesting that quantum-group descriptions are often available [5, 6, 7]; in particular, in Ref. [7] it was shown that the relations (1.1)-(1.2) can be associated to $S U_{q}(n)$ covariance.

The minimum-length scenario (1.1) would already require that the conceptual framework of Quantum Gravity be significantly different from the one of "ordinary" (non-gravitational) Quantum Mechanics']. However, as emphasized in Ref. [9], even more dramatic consequences for the measurability of distances appear to emerge from the analysis of additional contributions to the uncertainty relations which are associated to the fact that the limit of "classical" (infinitely massive) devices might not be accessible in Quantum Gravity. In particular, the analysis in Ref. [9] has led to the proposal of an alternative to the bound (1.1). By taking into account both the quantum nature of the agents involved in the measurement and the gravitational effects associated to the devices, one finds [9] that the measurability of distances is bound by a quantity that (as needed for the decoherence mechanism discussed in Ref. [10]) grows with the time required by the measurement procedure

$$
\min [\Delta L] \sim l_{p} \sqrt{\frac{c T}{s}} \sim l_{p} \sqrt{\frac{L}{s}},
$$

where $L$ is the distance being measured, $s$ is a length scale characterizing the spatial extension of the devices (e.g., clocks) used in the measurement, $T$ is the time needed to complete the procedure of measuring $L$, and on the right-hand-side we used the fact that, assuming the measurement procedure uses massless probes, one has typically $T \sim L$. Notice that for all acceptable values [9] of $s$ (i.e. $s<L$ ) the bound ([1.3) is more stringent than (1.1); this is a direct consequence of the fact that the analyses leading to (1.1) had implicitly relied on the availability of ideal classical devices in the measurement procedure.

While, as mentioned above, critical string theories provide a framework for the bound (1.1), it appears that noncritical string theories might provide a framework for the bound (1.3). In particular, within "Liouville" noncritical string theories, in which the target time is identified with the Liouville mode [11], the nature of the dynamics of the light probes exchanged in a typical procedure of measurement of a distance was shown [12] to lead to a measurability bound of type (1.3).

\footnotetext{
${ }^{3}$ In particular, in Ref. [8] it was shown that when gravitational effects are taken into account in a (quantum) measurement process then the masses of the probes used in the measurement induce a change in the space-time metric and this is associated to the emergence of nonlocality. The nature of this gravitationally-induced nonlocality suggests [8] a modification of the fundamental commutators.
} 
An interesting problem is the one of finding a quantum-group (and quantum-Liealgebra) framework for (1.3), just like Ref. [7] has provided a quantum-group framework for (1.1)-(1.2). The notion of quantum group as a Hopf algebra permits to consider deformed symmetries; in fact, the Hopf algebra axioms provide simultaneously an algebraic generalization of the definition of Lie group as well as of Lie algebra. As exemplified by the formulae in the following section, the phase space containing the coordinate and momentum sectors can be described in the quantum-deformed case as a semidirect product of two dual Hopf algebras describing the coordinates and momentum sectors. Such a definition of quantum phase space has been first proposed by Majid [5], and it is endoved with the property that in the undeformed case (coordinates and momenta described by Abelian Hopf algebra with primitive coproducts) one obtains the standard quantum mechanical Heisenberg commutation relations.t The so-called $\kappa$-deformations [14, 15, 16, 17] provide an example of this type of quantum deformations of relativistic symmetries, and one of us recently argued [18] that $\kappa$-deformed symmetries might provide an algebraic abstraction of the measurability bound (1.3). The analysis reported in 18 was somewhat preliminary since only the coordinate sector was considered, but the bound (1.3) emerged rather compellingly, as a direct consequence of the noncommuting space-time coordinates of $\kappa$-deformed Minkowski space. Encouraged by the findings of Ref. [18], in this paper we explore further the relation between $\kappa$-Poincaré and (1.3); specifically, we extend the analysis of Ref. [18] from the confines of the space-time coordinate sector to the full structure of the $\kappa$-deformed phase space. We primarily consider the $\kappa$-deformed Poincaré symmetries in the bicrossproduct basis [5, 16], which appears to be a very natural framework for the quantum deformations of semidirect product algebras, and outside of the coordinate sector we identify two structures which could affect the analysis of Ref. [18]: the $\kappa$-deformed mass-shell condition, which is associated to the Casimir and suggests a modification of the propagation of the light probes exchanged during measurement, and the nontrivial commutation relation between three-momenta and quantum time coordinate, which we find to have important consequences for the analysis of the propagation of heavy probes exchanged during measurement. As discussed below, our analysis uncovers new nonnegligible contributions to the bound on the measurability of distances. These contributions are however comparable to the one identified in Ref. 18\|, and therefore the order of magnitude of the effect discussed in Ref. 18 is confirmed by our analysis. These findings provide additional evidence of a relation between $\kappa$-Poincaré and the bound (1.3), and thereby contribute to the development of a physical interpretation of this class of deformations. We hope that this will provide further motivation for experimentalists to investigate the theoretical framework here advocated, especially exploiting the recent remarkable discoveries [19] in the phenomenology of gamma-ray bursts that allow [20] a direct test of some of the predictions of the $\kappa$ deformations of Poincaré symmetries.

\footnotetext{
${ }^{4}$ In the literature sometimes the semidirect product construction for two dual Hopf algebras describing respectively quantum Lie group and quantum Lie algebra is called "Heisenberg double" (see, e.g., Ref. 13]).

${ }^{5}$ Previous analyses attempting to bound the dimensionful parameter $\kappa$ only probed values of $\kappa$ that were several orderds of magnitude below the Planck scale (see, e.g., Ref. [12, 21, 22]), but now that we have definitive evidence that gamma-ray bursts are at cosmological distances we can expect [20] to probe values of $\kappa$ all the way up to the Planck scale.
} 


\section{$2 \kappa$-deformed quantum relativistic phase space}

The standard form of the covariant fourdimensional Heisenberg commutation relations, describing quantum-mechanical covariant phase space looks as follows:

$$
\left[x_{\mu}, p_{\nu}\right]=i \hbar g_{\mu \nu}, \quad g_{\mu \nu}=\operatorname{diag}(-1,1,1,1) .
$$

The space-time coordinates $x_{\mu}(\mu=0,1,2,3)$ can be identified with the translation sector of the Poincaré group, and the fourmomenta $p_{\mu}(\mu=0,1,2,3)$ are given by the translation generators of the Poincaré algebra. In considering quantum deformations of relativistic symmetries as describing the modification of space-time structure one is lead to the study of the possible quantum Poincaré groups. fo The classification of quantum deformations of $D=4$ Poincaré groups in the framework of Hopf algebras was given by Podleś and Woronowicz ([25]; see also [26]) and provides the most general class of noncommutative space-time coordinates $\hat{x}_{\nu}$ allowed by the quantum-group formalism. If we assume that the quantum deformation does not affect the nonrelativistic kinematics, i.e. we preserve the nonrelativistic $O(3)$ rotations classical and $O(3)$ covariance, the only consistent class of noncommuting space-time coordinates is described by the relations of the $\kappa$-deformed Minkowski space with commuting classical space coordinates. In order to describe the relativistic phase space we start with the deformed Hopf algebra of fourmomenta $\hat{p}_{\mu}$ written as follows

$$
\begin{aligned}
{\left[\hat{p}_{0}, \hat{p}_{k}\right] } & =0 \\
\Delta\left(\hat{p}_{0}\right) & =\hat{p}_{0} \otimes 1+1 \otimes \hat{p}_{0} \\
\Delta\left(\hat{p}_{k}\right) & =\hat{p}_{k} \otimes e^{\alpha \hat{p}_{0}}+e^{\beta \hat{p}_{0}} \otimes \hat{p}_{k}
\end{aligned}
$$

with antipode and counit given by

$$
S\left(\hat{p}_{k}\right)=-e^{-(\alpha+\beta) \hat{p}_{0}} \hat{p}_{k} \quad S\left(\hat{p}_{0}\right)=-\hat{p}_{0} \quad \epsilon\left(\hat{p}_{\mu}\right)=0 .
$$

Using the duality relations involving the fundamental constant $\hbar$ (Planck's constant)

$$
\left\langle\hat{x}_{\mu}, \hat{p}_{\nu}\right\rangle=-i \hbar g_{\mu \nu} \quad g_{\mu \nu}=(-1,1,1,1)
$$

we obtain the noncommutative deformed configuration space $\mathcal{X}$ as a Hopf algebra with the following algebra and coalgebra structure

$$
\left[\hat{x}_{0}, \hat{x}_{k}\right]=i \hbar(\beta-\alpha) \hat{x}_{k}, \quad\left[\hat{x}_{k}, \hat{x}_{l}\right]=0
$$

\footnotetext{
${ }^{6}$ We take into consideration here only the genuine 10 -generator quantum deformations of $D=4$ Poincaré symmetries. In particular, the "standard" $q$-deformations are not considered. These $q$ deformations require adding an eleventh (dilatation) generator, i.e. one deals with the dilatation extended Poincaré algebra [23]. In such a case the corresponding quantum phase space is much more complicated (see, e.g., 24]), and the deformation parameter is dimensionless, rendering difficult the physical separation between the ordinary regime of commutative space-time coordinates and the shortdistance regime in which non-commutativity sets in.
} 


$$
\begin{aligned}
\Delta\left(\hat{x}_{\mu}\right) & =\hat{x}_{\mu} \otimes 1+1 \otimes \hat{x}_{\mu}, \\
S\left(\hat{x}_{\mu}\right) & =-\hat{x}_{\mu}, \quad \epsilon\left(\hat{x}_{\mu}\right)=0 .
\end{aligned}
$$

The deformed phase space can be considered as the vector space $\mathcal{X} \otimes \mathcal{P}$ with the product (see [5])

$$
(x \otimes p)(\tilde{x} \otimes \tilde{p})=x\left(p_{(1)} \triangleright \tilde{x}\right) \otimes p_{(2)} \tilde{p}
$$

where left action is given by

$$
p \triangleright x=\left\langle p, x_{(2)}\right\rangle x_{(1)}
$$

The product $(2.8)$ can be rewritten as the commutators between coordinates and momenta by using the obvious isomorphism $x \sim x \otimes 1, p \sim 1 \otimes p$. This procedure provides the following commutation relations (see also [27, 28])

$$
\begin{array}{rlrl}
{\left[\hat{x}_{k}, \hat{p}_{l}\right]} & =i \hbar \delta_{k l} e^{\alpha \hat{p}_{0}}, & & {\left[\hat{x}_{k}, \hat{p}_{0}\right]=0,} \\
{\left[\hat{x}_{0}, \hat{p}_{k}\right]=-i \hbar \beta \hat{p}_{k},} & {\left[\hat{x}_{0}, \hat{p}_{0}\right]=-i \hbar .}
\end{array}
$$

The set of relations (2.2), (2.6) and (2.10) describes the deformed relativistic quantum phase space.

Introducing the dispersion of the observable $a$ in quantum mechanical sense by

$$
\Delta(a)=\sqrt{\left\langle a^{2}\right\rangle-\langle a\rangle^{2}}
$$

we have

$$
\Delta(a) \Delta(b) \geq \frac{1}{2}|\langle c\rangle| \quad \text { where } \quad c=[a, b]
$$

We obtain deformed uncertainty relations in the form

$$
\begin{aligned}
& \Delta \hat{x}_{0} \Delta \hat{x}_{k} \geq \frac{\hbar}{2}|(\beta-\alpha)|\left|\left\langle\hat{x}_{k}\right\rangle\right| \\
& \Delta \hat{p}_{k} \Delta \hat{x}_{l} \geq \frac{\hbar}{2} \delta_{k l}\left\langle e^{\alpha \hat{p}_{0}}\right\rangle \\
& \Delta \hat{p}_{0} \Delta \hat{x}_{0} \geq \frac{\hbar}{2} \\
& \Delta \hat{p}_{k} \Delta \hat{x}_{0} \geq \frac{\hbar}{2}\left|\left\langle\beta \hat{p}_{k}\right\rangle\right|
\end{aligned}
$$

Depending on a choice of the parameters $\alpha$ and $\beta$ we can distinguish the following cases ( $c$-speed of light, $\kappa$-(mass like) deformation parameter):

i) $\alpha=\beta=0 \quad$; standard form of nondeformed covariant phase space,

ii) $\alpha=\beta$; trivially deformed phase space with commuting configuration space,

iii) $\alpha=-\beta=\frac{1}{2 \kappa c} ; \kappa$-deformed phase space in the standard basis (see 29]),

iv) $\alpha=0, \beta=-\frac{1}{\kappa c} \quad ; \quad \kappa$-deformed phase space in the bicrossproduct basis (see [16]), 
v) $\alpha=\frac{1}{\kappa c}, \beta=0 \quad ; \quad \kappa$-deformed phase space in the bicrossproduct basis (see [17]), vi) $\alpha=0, \beta=\frac{1}{\kappa c} \quad ; \quad \kappa$-deformed phase space in the bicrossproduct basis (the case (v)) with transposed coproduct.

The Quantum-Gravity arguments advocated in the next sections make contact with the bicrossproduct basis, and therefore (also for definiteness) in the following we focus on the case (vi). The set of relations (2.2), (2.6) and (2.10) for our choice of parameters (vi) are the following

$$
\begin{aligned}
{\left[\hat{p}_{0}, \hat{p}_{k}\right] } & =0 & & \\
{\left[\hat{x}_{0}, \hat{x}_{k}\right] } & =\frac{i \hbar}{\kappa c} \hat{x}_{k}, & & {\left[\hat{x}_{k}, \hat{x}_{l}\right]=0 } \\
{\left[\hat{x}_{k}, \hat{p}_{l}\right] } & =i \hbar \delta_{k l}, & & {\left[\hat{x}_{k}, \hat{p}_{0}\right]=0 } \\
{\left[\hat{x}_{0}, \hat{p}_{k}\right] } & =-\frac{i \hbar}{\kappa c} \hat{p}_{k}, & & {\left[\hat{x}_{0}, \hat{p}_{0}\right]=-i \hbar }
\end{aligned}
$$

and are $\kappa$-Poincaré covariant 円.

The modified covariant Heisenberg uncertainty relations follow from the relations (2.14), therefore we obtain $\kappa$-deformed uncertainty relations

$$
\begin{aligned}
\Delta \hat{t} \Delta \hat{x}_{k} & \geq \frac{\hbar}{2 \kappa c^{2}}\left|\left\langle\hat{x}_{k}\right\rangle\right|=\frac{1}{2} \frac{l_{\kappa}}{c}\left|\left\langle\hat{x}_{k}\right\rangle\right|, \\
\Delta \hat{p}_{k} \Delta \hat{x}_{l} & \geq \frac{1}{2} \hbar \delta_{k l}, \\
\Delta \hat{E} \Delta \hat{t} & \geq \frac{1}{2} \hbar \\
\Delta \hat{p}_{k} \Delta \hat{t} & \geq \frac{\hbar}{2 \kappa c^{2}}\left|\left\langle\hat{p}_{k}\right\rangle\right|=\frac{1}{2} \frac{l_{\kappa}}{c}\left|\left\langle\hat{p}_{k}\right\rangle\right| .
\end{aligned}
$$

where $l_{\kappa}=\frac{\hbar}{\kappa c}$ describes the fundamental length at which the time variable should already be considered noncommutative. In comparison with the discussion in Ref. 18, which only considered the coordinate sector, the significant new element that emerged in our present analysis is the relation $(2.15 \mathrm{~d})$. Interestingly, multiplying the three relations (2.15a), (2.15b) and (2.15d) one obtains

$$
(\Delta \hat{t})^{2}\left(\Delta \hat{x}_{l} \Delta \hat{p}_{l}\right)^{2} \geq \frac{\hbar}{8} \frac{l_{\kappa}^{2}}{c^{2}}\left|\left\langle\hat{x}_{l}\right\rangle\left\langle\hat{p}_{l}\right\rangle\right|
$$

(where no sum over the index $l$ is to be understood). This suggests that a wave packet with minimal standard $(\Delta x \Delta p)$ uncertainty has the largest uncertainty in the localization of time. (In ordinary quantum mechanics $l_{\kappa}=0$ and there is no such correlation.)

It is also interesting to consider the relation $(2.15 \mathrm{~d})$ under the assumption that the three-momenta $\hat{p}_{k}$ can be expressed by a general formula $\hat{p}_{i}=\mathcal{M}\left(v^{2}\right) v_{i}$, in which case

\footnotetext{
${ }^{7}$ The $\kappa$-covariance of the relations $(2.14 \mathrm{~b})$ has been shown firstly in Ref. 16. The $\kappa$-covariance of the whole quantum $\kappa$-deformed Heisenberg algebra follows from the general properties of the semidirect product, defined by the relations $(2.14 \mathrm{~b})$ and $(2.14 \mathrm{c}-\mathrm{d})$. (see, e.g., 30])
} 
$\Delta \hat{p}_{i}=\mathcal{M}_{i j} \Delta v_{k}$ with $\mathcal{M}_{i j}=\mathcal{M}\left[\delta_{i j}+2 v_{i} v_{j}(\ln \mathcal{M})^{\prime}\right]$. Then $(2.15 \mathrm{~d})$ implies

$$
\Delta \hat{t} \Delta v_{i} \geq \frac{l_{\kappa}}{c} \mathcal{M}(v) \mathcal{M}_{i j}^{-1}(v) v_{j}
$$

Because in part of our measurement analysis we shall consider light probes, we now discuss the modification of the kinematics of $\kappa$-deformed photons. We shall assume that the generators of the $\kappa$-deformed Poincaré algebra in bicrossproduct basis describes the "physical" generators of space-time symmetries. In the bicrossproduct basis the $\kappa$ deformed mass Casimir takes the form

$$
C_{2}^{\kappa}=\frac{1}{c^{2}} \vec{P}^{2} e^{-\frac{P_{0}}{\kappa c}}-\left(2 \kappa \sinh \frac{P_{0}}{2 \kappa c}\right)^{2}=-M^{2},
$$

where $P_{\mu}$ are the generators of space-time translations and $M$ denotes the $\kappa$-invariant mass parameter. For $M=0$ ( $\kappa$-deformed photons) from (2.18) one obtains that (we identify $\left.P_{\mu} \equiv \hat{p}_{\mu}\right)$

$$
\hat{p}_{0}=\kappa c \ln \left(1+\frac{|\overrightarrow{\hat{p}}|}{\kappa c}\right)=|\overrightarrow{\hat{p}}|-\frac{|\overrightarrow{\hat{p}}|^{2}}{2 \kappa c}+O\left(\frac{1}{\kappa^{2}}\right)
$$

and in particular the velocity formula for massless $\kappa$-deformed quanta looks as follows 8 $\left(E=c \hat{p}_{0}\right)$

$$
v_{i}=\frac{\partial E}{\partial \hat{p}_{i}}=\frac{c}{1+\frac{|\overrightarrow{\hat{p}}|}{\kappa c}} \frac{\hat{p}_{i}}{|\overrightarrow{\hat{p}}|}
$$

or

$$
v=|\vec{v}|=\frac{c}{1+\frac{|\overrightarrow{\hat{p}}|}{\kappa c}}=c-\frac{|\overrightarrow{\hat{p}}|}{\kappa}+O\left(\frac{1}{\kappa^{2}}\right)
$$

The inverse formula, which can be inserted in (2.17), looks as follows

$$
\hat{p}_{i}=\kappa \frac{c}{v}\left(\frac{c}{v}-1\right) v_{i}
$$

and it is linear in the deformation parameter $\kappa$.

\section{Measurement of distance and covariant $\kappa$-deformed phase space}

In this section we analyze the measurement of the distance $L$ between two bodies as it results from a plausible physical interpretation of the uncertainty relations (2.15a)(2.15d). Like the related studies [9, 10, 12] we consider the procedure of measurement of distances set out by Wigner [31], which relies on the exchange of a probe/signal between the bodies. The distance is therefore measured as $L=v T / 2$, where $v$ is the velocity of the probe and $T$ is the time (being measured by a clock) spent by the probe to go from one body to the other and return. In general the quantum mechanical nature of

${ }^{8}$ The relation (2.20a) is valid as a consequence of the Hamiltonian equation of motion $\dot{x}_{i}=\partial H / \partial p_{i}-$ $\left(x_{i} / \kappa\right) \partial H / \partial x_{0}$. [See Ref. [17], Eq. (4.22).] For the $\kappa$-photon here considered, since $H=H\left(p_{i}\right)$, the velocities are classical $\left(\left[v_{i}, v_{j}\right]=0\right)$. 
the agents intervening in the experiment introduces uncertainties in the measurement of $L$, and in particular one finds that 9

$$
\Delta L \geq[\Delta L]_{\text {clock }}+[\Delta L]_{\text {probe }},
$$

i.e. the uncertainty in the measurement of $L$ receives of course contributions that originate from the quantum mechanical nature of the "clock" (the timing/triggering device employed in the measurement) and from the quantum mechanical nature of the probe exchanged between the bodies. A significant contribution to $\Delta L_{\text {clock }}$ was uncovered in Ref. [9]; this results in the relation

$$
[\Delta L]_{\text {clock }} \geq l_{p} \sqrt{\frac{c T}{s}}
$$

where $s$ is a length scale characterizing the spatial extension of the clock (e.g., the radius of a spherically-symmetric clock) and $T$ is the time needed to complete the procedure of measuring $L$ (i.e. $T$ is the time that the clock measures).

Within ordinary quantum mechanics the quantum mechanical nature of the probe (while contributing in general to the uncertainty) does not contribute to the bound on the measurability of $L$ (i.e. a suitable measurement set up can be found so that the quantum mechanical nature of the probe does not lead to a contribution to $\Delta L$ ). It was shown in Ref. 18] that instead the kinematics of quantum $\kappa$-Minkowski space-time does lead to a nontrivial $[\Delta L]_{\text {probe }}$, and interestingly this turns out to be of the same form of the $[\Delta L]_{\text {clock }}$ in (3.2). As announced in the Introduction we are interested in extending the analysis of Ref. [18] to include structure from the full $\kappa$-deformed phase space. We are also more general than Ref.[18] and other related work (see, e.g., Ref. [9, 10, 12]) in that we not only consider massless particles as the probes exchanged in the Wigner measurement, but we also consider the opposite limit in which the probes are ultraheavy.

\subsection{Using a heavy probe}

In general combining the contribution (3.2), which originates from the quantum mechanical nature of the clock, with uncertainties due to the quantum mechanical nature of the probe one finds that

$$
\Delta L \geq l_{p} \sqrt{\frac{c T}{s}}+\Delta x+v \Delta t+T \Delta v
$$

where $\Delta x$ and $\Delta t$ are the uncertainties on the space-time position $\square$ of the probe at the "final time" $T$, while $\Delta v$ is the uncertainty on the velocity of the probe.

\footnotetext{
${ }^{9}$ Of course there are other contributions to $\Delta L$ (e.g., coming from the quantum mechanical nature of the other devices used in the experiment [9]); however, since they obviously contribute additively to the total uncertainty in the measurement of $L$, these uncertainties could only make stronger the bound derived in the following.

${ }^{10}$ As implicit in the terminology here adopted, the Wigner measurement procedure is essentially one-dimensional, and the only relevant spatial coordinate is the one along the axis passing through the bodies whose distance is being measured.
} 
The first contribution on the right-hand-side of (3.3) originates from the quantum mechanical nature of the clock, and it is interesting to notice that in the case of a heavy probe the proportionality to $\sqrt{T}$ of that term, which always signals decoherence effects (e.g., the more time goes by, the more the quantum clock decoheres according to the ideas in Refs. [9, 10]), can be turned into a proportionality to $\sqrt{L / v}$, i.e. the uncertainty actually diverges in the limit of vanishing velocity as expected in a context involving decoherence (small velocities imply large times).

Concerning the contributions on the right-hand-side of (3.3) that originate from the quantum mechanical nature of the probe, it is interesting to observe that in ordinary quantum mechanics $\Delta x, \Delta t$ and $\Delta v$ are not correlated and therefore they do not lead to a contribution to the bound on the measurability of $L$. However, the $\kappa$ deformation induces correlations between $\Delta x, \Delta t$ and $\Delta v$. In particular, we observe that (2.15a)(2.15d) imply (for an ideal heavy/nonrelativistic probe with $p=M v$ and interpreting the $x$ on the right-hand-side of (2.15a) as the distance traveled by the probe)

$$
\Delta v \geq \frac{l_{\kappa} v}{2 c \Delta t}
$$

and

$$
\Delta x \geq \frac{l_{\kappa} L}{2 c \Delta t} .
$$

This relations together with the fact that $v \sim L / T$ allow to rewrite (3.3) as

$$
\Delta L \geq l_{p} \sqrt{\frac{c T}{s}}+\frac{l_{\kappa} L}{2 c \Delta t}+\frac{L}{T} \Delta t+\frac{l_{\kappa} L}{2 c \Delta t} .
$$

This uncertainty can be minimized by preparing the probe in a state with $v \sim c l_{p} / \sqrt{s l_{\kappa}}$, i.e. $T \sim L \sqrt{s l_{\kappa}} /\left(c l_{p}\right)$, and $\Delta t \sim \sqrt{l_{\kappa} T / c}$, and this results in the measurability bound

$$
\min [\Delta L] \sim \sqrt{L l_{p} \sqrt{\frac{l_{\kappa}}{s}}}
$$

The fact that this bound emerging from our analysis of Wigner measurement using a heavy probe manifests the same $\sqrt{L}$ behavior encountered in the heuristic quantumgravity analysis of the clock involved in the measurement is a rather interesting aspect of the covariantly $\kappa$-deformed phase space. In fact, Eq.(3.4), which reflects the specific structure of the $\kappa$-deformed commutation relation between three-momenta and quantum time coordinate, plays a nontrivial role in establishing that the $\kappa$-deformed kinematics of the heavy probe leads to an uncertainty with this $\sqrt{L}$ behavior.

\subsection{Using a massless probe}

Of course, also in the case of a Wigner measurement involving a massless probe one finds that

$$
\Delta L \geq l_{p} \sqrt{\frac{c T}{s}}+\Delta x+c \Delta t+T \Delta v,
$$


and again the $\kappa$ deformation induces correlations between $\Delta x, \Delta t$ and $\Delta v$. In particular, concerning the correlation between $\Delta x$ and $\Delta t$ using again (2.15a) one finds

$$
\Delta t \geq \frac{\hbar L}{2 \kappa c^{2} \Delta x}
$$

Moreover, if the probe is massless with modified velocity (2.20b) one finds that

$$
\Delta v \sim \frac{\Delta P}{\kappa} \sim \frac{\hbar}{2 \kappa \Delta x}
$$

where on the right-hand-side we used (2.15b).

Using (3.9) and (3.10) one can rewrite (3.8) as

$$
\Delta L \geq l_{p} \sqrt{\frac{c T}{s}}+\Delta x+\frac{\hbar L}{2 \kappa c \Delta x}+\frac{\hbar T}{2 \kappa \Delta x},
$$

and therefore, also taking into account that $L \sim c T / 2$ and $l_{\kappa} \equiv \hbar /(\kappa c)$, one finds that the minimal value of $\Delta L$ is obtained if $(\Delta x)^{2} \sim L l_{\kappa}$ and this implies that the minimal uncertainty in the measurement of the distance $L$ is

$$
\min [\Delta L] \sim \sqrt{\frac{L l_{p}^{2}}{s}}+\sqrt{L l_{\kappa}}
$$

Again we find the $\sqrt{L}$ behavior, and again the full structure of the covariantly $\kappa$ deformed phase space advocated here plays a rather central role in obtaining this result; in fact, the relation (2.18) ensures that the fourth term on the right-hand side of Eq. (3.8) (which was not considered in Ref. [18]) is of the same order as the second term on the right-hand side of Eq. (3.8), which is the one considered in Ref. [18.

While the $\sqrt{L}$ behavior is of course the most robust outcome of these analyses, it is interesting to notice the interplay between the scale $l_{\kappa}$, which characterizes the $\kappa$ deformation, and the scales $s$ and $l_{p}$, which characterize heuristic quantum-gravity arguments. Although the relative magnitude of these scales could only be determined once a full Quantum Gravity formalism became available, it is quite natural to guess that if $\kappa$ deformations were to have physical applications it might be that $l_{\kappa} \sim l_{p}$. Moreover, from the role of $s$ in the measurement procedure it is clear [9, 18] that $s \geq l_{p}$, and since the measurability bound should be a general property of the theory it is conceivable that also $s \sim l_{p}$. This, for example, appears to fit rather well the schemes, such as the one discussed in Ref. [32], in which "fundamental clocks" are intrinsic to the formulation of the quantum-gravity approach. For $l_{\kappa} \sim l_{p} \sim s$ the heavy probe and the massless probe considered in this and in the previous subsection lead to exactly

\footnotetext{
${ }^{11}$ It is interesting to notice that $\kappa$-deformed mass-shell condition and $\kappa$-commutation relation between three-momenta and quantum time coordinate are somewhat related. In fact, for a minimumuncertainty state in the framework of $\kappa$-deformed kinematics one has $\Delta E \Delta t \sim \hbar / 2$ and $\Delta p \Delta t \sim$ $l_{\kappa} p /(2 c)$, and this is consistent with a given dispersion relation $E(p)$ only if $E(p) \sim\left(c \hbar / l_{\kappa}\right) \ln \left(p / p^{*}\right)$ (with $p^{*}$ a constant to be otherwise determined) which coincides with the asymptotic behavior of the $\kappa$-deformed dispersion relation (see (2.19)).
} 
(up to an overall numerical factor of order 1) the same bound in the context of the Wigner measurement, and even the heuristic quantum-gravity measurement analysis of Ref. [9] reproduces this bound exactly (again up to an overall numerical factor of order 1). Nevertheless, especially in light of the fact that very little will be known about $s$ until a fully consistent (and genuinely quantum) theory of gravity is available, it is interesting to observe that if $s \neq l_{p}$ (i.e. $s>l_{p}$ ) the Wigner measurement using a heavy probe is actually a "better measurement" (weaker bound) than its counterpart using a massless probe. Since most of the previous studies of quantum-gravity measurability bounds have relied on massless probes, our results suggest that a reanalysis of those studies might be necessary.

\section{Closing Remarks}

The covariant $\kappa$-deformation of relativistic symmetries here considered, and the associated covariant $\kappa$-deformation of the Heisenberg algebra (2.14), has several appealing properties as a candidate for the high-energy modification of classical relativistic symmetries. It provides a rather moderate (at least in comparison with some of its alternatives) deformation of classical relativistic symmetries, which in particular reflects the reasonable expectation that, if any of the space-time coordinates is to be special, the special coordinate should be time. (Interestingly this intuition appears to be also realized in certain approaches to string theory, see e.g. Ref. [11.) As manifest in the relations (2.15a)-(2.15d), the $\kappa$-modifications of the covariant Heisenberg commutations relations are of quantum mechanical nature, i.e. proportional to the Planck constant $\hbar$. This suggests that the $\kappa$-deformation can be related with the quantum corrections to the classical dynamics of the space-time geometry. In extending the analysis of Ref. [18] from the space-time coordinate sector to the full structure of the $\kappa$-deformed phase space, our analysis has provided additional evidence that the bounds on the measurability of distances associated with the uncertainty relations characterizing the $\kappa$-deformed covariant Heisenberg algebra (2.14) are consistent with independent heuristic quantum-gravity analyses of such measurability bounds. Based on this consistency between heuristic quantum-gravity measurability analysis and $\kappa$-Poincaré measurability analysis one is prompted to consider the possibility that [18, 33 at length scales larger than the Planck length (but of course smaller than the length scales already probed experimentally) the $\kappa$-deformations of Poincaré symmetries might play a role in the effective description of Quantum Gravity. This is not completely surprising since some of the most popular Quantum-Gravity scenarios, such as the ones based on a spacetime lattice and the ones involving a foamy Quantum-Gravity vacuum, would plausibly lead [33 to deformations of Poincaré symmetries, and, in particular, the analysis of Ref. [12 appears to suggest that propagation in a foamy Quantum Gravity vacuum might be characterized by a $\kappa$-deformed dispersion relation.

The three-momentum-dependent (i.e. energy-dependent) "speed of light" (2.20b) is a novel phenomenon that arises in the framework here considered. As mentioned, it has the same functional form (upon appropriate identification between $\kappa$ and the string scale) as the energy-dependent speed of light recently discussed [12] in the non-critical ("Liouville") string literature. Both in the $\kappa$-Poincaré and in the string theory contexts the deviation from ordinary physics, while very significant at the conceptual level, is 
rather marginal from the phenomenological viewpoint. For example, for photons of energies of order $1 \mathrm{GeV}$ the Eq. (2.20b) entails a minuscule $10^{-19} c$ correction with respect to the ordinary scenario with constant speed of light. At least when $\kappa$ is identified with the Planck scale, the Eq. (2.20B) is completely consistent with presently available experimental data 112, 21, 22. However (as already emphasized in Refs. 20, 34] and references therein), some of the modern techniques of investigation of astrophysical phenomena could soon bring remarkable progress in the investigation of space-time symmetries. In our case the best laboratory appears to be provided by gamma-ray bursts, and, now that there is convincing evidence 19 that these bursts have cosmological origin, we can expect [20] that within a few years gamma-ray-burst data will test conclusively the $\kappa$ deformations we considered.

\section{References}

1. C.A. Mead, Phys. Rev. 135B (1964) 849.

2. See, e.g., T. Padmanabhan, Class. Quantum Grav. 4 (1987) L107, and the recent review L.J. Garay, Int. J. Mod. Phys. A10 (1995) 145.

3. S. Dopplicher, K. Fredenhagen, and J.E. Roberts, Phys. Lett. B331 (1994) 39; Comm. Math. Phys. 172 (1995) 187.

4. G. Veneziano, Europhys. Lett. 2 (1986) 199; D.J. Gross and P.F. Mende, Nucl. Phys. B303 (1988) 407; D. Amati, M. Ciafaloni, G. Veneziano, Phys. Lett. B216 (1989) 41; K. Konishi, G. Paffuti, P. Provero, Phys. Lett. B234 (1990) 276; T. Yoneya, Mod. Phys. Lett. A4, 1587 (1989); Phys. Rev. Lett. 78, 1219 (1997).

5. S. Majid, Class. Quant. Grav. 5 (1987) 1587; "Foundations of Quantum Groups" (Cambridge Univ. Press., 1995).

6. M. Maggiore, Phys. Lett. B304 (1993) 65.

7. A. Kempf, J. Math. Phys. 35 (1994) 4483; A. Kempf, G. Mangano, and R.B. Mann, Phys. Rev. D52 (1995) 1108; A. Kempf and G. Mangano, Phys. Rev. D55 (1997) 7909.

8. D.V. Ahluwalia, Phys. Lett. B339 (1994) 301.

9. G. Amelino-Camelia, Mod. Phys. Lett. A9 (1994) 3415; A11 (1996) 1411.

10. F. Karolyhazy, Il Nuovo Cimento A42 (1966) 390; L. Diosi, B. Lukacs, Phys. Lett. A142 (1989) 331; Y.J. Ng and H. Van Dam, Mod. Phys. Lett. A9 (1994) 335; A10 (1995) 2801.

11. J. Ellis, N.E. Mavromatos, and D.V. Nanopoulos, Phys. Lett. B293 (1992) 37; Mod. Phys. Lett. A10 (1995) 425.

12. G. Amelino-Camelia, J. Ellis, N.E. Mavromatos and V.D. Nanopoulos, Int. Journ. Mod. Phys. A12 (1997) 607

13. A.Yu. Alekseev, L.D. Faddeev, Comm. Math. Phys. 141 (1991) 413. 
14. J. Lukierski, A. Nowicki, H. Ruegg and V.N. Tolstoy, Phys. Lett. B264 (1991) 331.

15. J. Lukierski, A. Nowicki and H. Ruegg, Phys. Lett. B293 (1992) 334.

16. S. Majid and H. Ruegg, Phys. Lett. B334 (1994) 348.

17. J. Lukierski, H. Ruegg and W.J. Zakrzewski, Ann. of Phys. 243 (1995) 90.

18. G. Amelino-Camelia, Phys. Lett. B392 (1997) 283.

19. See R. Wijers, Nature 393 (1998) 13, and references therein.

20. G. Amelino-Camelia, J. Ellis, N.E. Mavromatos, D.V. Nanopoulos, and S. Sarkar, Nature 393 (1998) 763.

21. G. Domokos and S. Kovesi-Domokos, Journ. Phys. G20 (1994) 1989.

22. J.P. Bowes and P.D. Jarvis, Class. Quant. Grav. 13 (1996) 1405.

23. S. Majid, Journ. Math. Phys. 34 (1993) 2045.

24. J. Schwenk and J. Wess Phys. Lett. B291 (1992) 273; M. Fichtmüller, A Lorek, and J. Wess hep-th/9511106.

25. P. Podleś, S.L. Woronowicz, Comm. Math. Phys. 178 (1996) 61.

26. P. Podleś, Comm. Math. Phys. 181 (1996) 569.

27. J. Lukierski, A. Nowicki, in "Quantum Group Symposium at GROUP21", ed. H.D. Doebner, V.K. Dobrev, Heron Press, Sofia 1997, p. 186; q-alg/9702003.

28. A. Nowicki, in "New Symmetries in the Theory of Fundamental Interactions", ed. J. Lukierski, M. Mozrzymas, PWN Warsaw 1997, p. 43; q-alg/9702004.

29. A. Nowicki, "Kappa Deformed Phase Space and Uncertainty Relations", math.QA/9803064.

30. J. Schupp, P. Watts and B. Zumino Comm. Math. Phys. 157 (1993) 305.

31. E.P. Wigner, Rev. Mod. Phys. 29 (1957) 41; H. Salecker, E.P. Wigner, Phys. Rev. 109 (1958) 571.

32. C. Rovelli, Class. Quantum Grav. 8 (1991) 297; ibid. 8 (1991) 317.

33. G. Amelino-Camelia, Mod. Phys. Lett. A13 (1998) 1319.

34. S.L. Glashow, Nucl. Phys. (Proc. Suppl.) B70 (1999) 180. 